\title{
TUBERCULOSIS DEL SENO
}

\author{
Dr. Hernando Amaya León*
}

Hace algún tiempo se planteó una posibilidad diagnóstica de tuberculosis en una paciente con lesión fistulizada del seno; todos sus exámenes fueron negativos a ese respecto, y a la postre resultó un exótico caso de saturnismo.

Surgieron por entonces interrogantes respecto a frecuencia, clínica y manejo de la Tuberculosis mamaria.

En los trabajos presentados sobre Tuberculosis Genital Femenina (1) no mencionamos este tema, y la literatura colombiana (2) no reporta ninguna investigación sobre el tópico.

La literatura mundial a nuestro alcance es muy escasa respecto a tuberculosis mamaria; en el Index Medicus y Excerpta Medica de los últimos 5 años, no hay nada relievante.

Al decir de Christopher (3), la lesión es muy rara; ésto es confirmado por todos los autores. Haagensen (4), escrutando 6.500 casos de lesiones del seno, entre 1938 y 1955 en Columbia University, encuentra 4 casos. Muir (5) en Escocia, relata 2 casos en 1.010 especímenes quirúrgicos del seno en 5 años. Norris (6) en la Universidad de Pensilvania aisló 1 caso entre 196 especímenes del seno. Boyd (7) en su texto, se limita a confirmar su extrema rareza, lo

mismo que Hosford (8), quien afirma que la enfermedad se ve tan solo en estados muy avanzados. Quizá los únicos que difieren de estos conceptos son los autores de Praxis Médica (9) quienes en 1960 dicen que la entidad "no es rara" constituyendo el $6 \%$ de las formas benignas de la fama.

Hemos intentado una revisión estadística en las principales entidades hospitalarias en Bogotá, cuya síntesis es la siguiente:

En los archivos del Hospital San Juan de Dios, entre 1952 y 1967 y sobre un total de casi 500.000 admisiones, figuran 3 casos; al ser revisadas esas historias clínicas, en dos se hizo la sugestión clínica sin confirmación posterior; y en una, correspondiendo a una enferma del servicio de Ginecología (1953), se diagnosticó por patología; buscando estas placas y revisadas con el jefe de Patología Ginecológica se confirmaron lesiones muy sospechosas de TB$C$, pero como en éllas no se investigó la presencia del bacilo de Koch, el diagnóstico se consideró no concluyente.

En la unidad de Cirugía General del mismo Hospital entre 146 casos

* Jefe del Departamento de Obstetricia y Ginecología Universidad Nacional. 
de lesiones del seno muy bien estudiadas entre 1965-1967 no se encontraron casos de tuberculosis.

En el Hospital Sanatorio Santa Clara, dedicado exclusivamente a tuberculosis, en la revisión estadística de casi 30 años de existencia, no hay reportado ni un solo caso de lesión mamaria tuberculosa.

En el Instituto Nacional de Cancerología, los siguientes datos me fueron facilitados por el patólogo $\mathrm{Dr}$. Martin:

\section{PATOLOGIA DE GLANDULA MAMARIA (1962-1966)}

Carcinoma de diferentes tópicos

Cistosarcoma filoides benignos y malignos

Enfermedad de Paget

Fibrosarcoma

Enfermedad de Hodgkin

Fibroadenoma

Papiloma Intracanalicular

Lipoma

Enfermedad fibroquística

Glándula mamaria supernumeraria

Hipertrofia e hiperplasia

Esteatonecrosis

Comedomastitis

Inflamación aguda y crónica no específica 89

Mastitis crónica granulomatosa no específica 6

(Nota: En el grupo de las mastitis crónicas granulomatosas no específicas se practicaron coloraciones de $Z$. N. siendo negativas para bacilos ácido alcohol resistentes).

Esta estadística es sin duda la más importante de las consultadas.

Con estos datos podemos concluir que la tuberculosis del seno es prácticamente desconocida en nuestro medio.

Clínica. Las descripciones en los textos son casi nulas respecto a esta entidad; vale la pena resaltar las de Julliard (10), quien en resumen afirma que la tuberculosis mamaria se descubre ocasionalmente en el embarazo o lactancia (esto no confirmado en nuestra experiencia) (11), es ge- neralmente unilateral y localizada en la región supero-externa.

Distingue 3 formas anatómicas: diseminada, confluente y pseudoneoplásica. Asegura que su sintomatología es muy pobre, que su coexistencia con cáncer es muy probable, y que el diagnóstico se basa en la presencia de núcleos tumorales y en lesiones fistulizadas, éstas en casos tardíos.

Diagnóstico. Una cosa es evidente a este respecto: si no se identifica el bacilo de Koch en las lesiones sospechosas, no puede establecerse el diagnóstico definitivo, ya que las lesiones patológicas aunque muy importantes no son confirmatorias.

Por este motivo, cuando se piense en tuberculosis del seno, debería enviarse parte del material en investigación al laboratorio especializado (Instituto Nacional de Salud en nuestra ciudad) para que ese material sea procesado y cultivado en orientación a identificar el bacilo tuberculoso. Estamos seguros que si esto se hiciera de rutina, encontraríamos algunos casos de tuberculosis mamaria, ya que el índice de prevalencia de la TBC pulmonar en nuestro medio es alto, y éste es el origen lógico de la localización en el seno.

Tratamiento. No hay normas precisas en los textos de estudio. Pero éstas podrían presumirse así:

a) Si el diagnóstico es preoperatorio, por biopsias y/o cultivos, se debe hacer tratamiento médico por los procedimientos usuales en la actualidad, a base de estreptomicina, isoniacida y PAS; a los 3-6 meses, intervenir la paciente y continuar luego el tratamiento médico hasta consolidación. A este respecto no podemos olvidar que la tuberculosis es enfermedad infecciosa de propagación ge- 
neralizada con afinidad específica por ciertos tejidos (entre los cuales no parece contarse el mamario); por tanto, su tratamiento debe ser similar no importa el sitio en donde se encuentre.

b) $\mathrm{Si}$ el diagnóstico es post-operatorio, para lo cual se recomienda incrementar el uso de cultivos del material quirúrgico, se debe practicar tratamiento intensivo a base de las drogas tuberculostáticas con las dosificaciones recomendadas en otras localizaciones.

\section{RESUMEN}

1. La tuberculosis mamaria es entidad muy rara.

2. En Bogotá es prácticamente desconocida.

3. Se recomienda incrementar el uso de cultivos en el material de biopsias y de piezas quirúrgicas para investigar el bacilo tuberculoso.

\section{BIBLIOGRAFIA}

1 AMAYA LEON H., VERGARA T. R., VILLAREAL M. J., y col. Tuberculosis Genital Femenina. Rev. Colomb. Obst. Ginec. XV: $111,1964$.

2 Asociación Colombiana de Facultades de Medicina. Indice de Literatura Médica Colombiana (1890-1960). Ed. Tercer Mundo, Bogotá. 1965.

3 CHRISTOPHER. Tratado de Patología Quirúrgica. Ed. Interamericana, p. 335, 1961.

4 HAAGENSEN C. D. Diseases of the Breast. W. B. Saunders Co., 1956.

5 MUIR'S. Textbook of Pathology. Ed. Arnold Ltd. London, 1960.

6 GOLDBERG B. Clinical Tuberculosis. II. J. 27. F. A. Davis Co., 1947.

7 BOYD W. A Textbook of Pathology. Lea and Febiger, p. 962, 1961.

8 HOSFORD J. Breast Infections. British Surg. Pract. II, p. 495. Butterworth Co. Ltd., London, 1951.

9 Praxis Medica. T. X. p. 10465. Ed. Latino-Amercianas, 1960.

10 JULLIARD A. Encyclopedie Medico Chirurgicale. Ginecologie Mamelle. p. 830 A 10.

11 AMAYA LEON H., FIOREZ A. J. Tuberculosis y Embarazo. Rev. Colomb. Obst. Ginec. XVI: 357, 1965. 